\title{
Liam Harte, Reading the Contemporary Irish Novel
} 1987-2007

\section{Sylvie Mikowski}

\section{Q OpenEdition}

1 Journals

\section{Édition électronique}

URL : http://journals.openedition.org/etudesirlandaises/4001

DOI : 10.4000/etudesirlandaises. 4001

ISSN : 2259-8863

Éditeur

Presses universitaires de Rennes

\section{Édition imprimée}

Date de publication : 20 novembre 2014

Pagination : 191-192

ISBN : 978-2-7535-3559-6

ISSN : 0183-973X

\section{Référence électronique}

Sylvie Mikowski, «Liam Harte, Reading the Contemporary Irish Novel 1987-2007 », Études irlandaises [En ligne], 39-2 | 2014, mis en ligne le 20 novembre 2014, consulté le 21 avril 2019. URL : http:// journals.openedition.org/etudesirlandaises/4001; DOI : 10.4000/etudesirlandaises.4001 
Liam Harte, Reading the Contemporary Irish Novel 1987-2007, Wiley Blackwell, Chichester, West Sussex, 2014, ISBN 978-1-4443-3620-7, 264 p.

Liam Harte, spécialiste reconnu et respecté du roman irlandais contemporain, propose ici la lecture approfondie de neuf romans, appartenant tous à l'ère pré-« tigre celtique ", soit de par leur date de publication, soit de par l'époque à laquelle leur action est située. Sur une période de vingt ans, l'auteur a retenu de la production romanesque irlandaise des œuvres déjà abondamment commentées, à tel point qu'on peut les considérer comme constitutives du canon contemporain. Liam Harte étudie ainsi sept écrivains hommes (Roddy Doyle, Patrick McCabe, John McGahern, Colm Toibin, Seamus Deane, Sebastian Barry), et deux femmes (Edna O'Brien et Anne Enright), chacun à travers l'analyse approfondie d'un seul de leurs romans (la trilogie de Barrytown pour Doyle), même si chaque chapitre commence par un court résumé de la vie et de la carrière des écrivains. Liam Harte a pu s'appuyer sur un nombre considérable de ressources critiques, mais il a su les synthétiser à merveille, et surtout dépasser cette somme en présentant des interprétations personnelles d'une pertinence et d'une lucidité toujours impressionnantes, exprimées dans une langue à la fois limpide, précise et extrêmement riche et élégante.

À certains égards, le prisme à travers lequel Harte considère ces vingt années de production romanesque en Irlande est à peu près le même que celui utilisé par nombre de ses prédécesseurs-parmi lesquels on peut citer les faiseurs d'opinion que sont devenus Declan Kiberd, Luke Gibbons, David Lloyd, Gerry Smyth ou encore Fintan O'Toole : celui de la relation entre histoire et fiction, du roman considéré comme miroir de la société, de l'Irlande vue comme une société postcoloniale encore accablée par le poids du passé, mais aussi des douloureux ajustements aux évolutions de la modernité. Certaines analyses font par ailleurs déjà l'objet d'un consensus critique : il en va ainsi de la présence du gothique dans The Butcher Boy, de l'écriture du traumatisme chez McGahern, du procès du conservatisme de l'Etat irlandais dans The Heather Blazing. Cependant même si Harte privilégie souvent une lecture historiciste des œuvres, suivant en cela la tendance critique dominante en Irlande, on remarquera qu'il a aussi fréquemment recours au vocabulaire de la psychanalyse, poussé dans ce sens par la prédominance des symptômes névrotiques propres à l'Irlande pré-« tigre celtique » dans les romans analysés, comme en atteste par exemple sa lecture du roman de Anne Enright The Gathering. Toujours pénétrant dans ses analyses et mettant en lumière des aspects parfois mal reconnus de certains textes - par exemple la dimension postcoloniale de Felicia's Journey -, Harte ne se départ pas pour autant d'un sens critique sévère, dénonçant par exemple les incohérences, voire ce qu'il considère comme les errances idéologiques, de Edna O’Brien dans House of Splendid Isola- 
tion, dans lequel le portrait du tueur de l'IRA est jugé complaisant. Harte n'hésite pas non plus à afficher des convictions autant esthétiques qu'idéologiques lorsqu'il condamne A Long Long Way de Sebastian Barry, dans lequel il reproche à l'auteur de susciter la compassion pour les soldats irlandais enrôlés pendant la Première Guerre mondiale, en développant ce qu'il appelle une « esthétique problématique et des aspects polémiques ». En Irlande, l'histoire, la politique et la littérature demeurent inextricablement mêlées.

Sylvie Mıкоwsкi

Laurence Cox, Buddhism and Ireland - From the Celts to the Counter-Culture and Beyond, Sheffield (UK)/Bristol (USA), Equinox, 2013, 413 p., ISBN 978-1908049-30-8.

Buddhism and Ireland propose l'exploration passionnante d'une question peu connue, l'histoire ou plutôt les histoires des interactions entre le bouddhisme et l'Irlande. Laurence Cox est sociologue, mais il offre au lecteur une réflexion interdisciplinaire de grande ampleur, un regard aux facettes multiples indispensable à l'évocation complète d'un sujet aussi complexe en perspective diachronique et synchronique. D'un point de vue théorique global, l'ambition de l'auteur est de montrer que l'on ne peut comprendre les phénomènes décrits que dans la perspective du système-monde. L'Irlande à différentes périodes est envisagée comme l'un des éléments d'espaces interdépendants qui définissent le cadre global de développements multiples. L'histoire du bouddhisme n'est donc pas une, mais plurielle; aucune continuité n'y est décelable, outre celle qui lie entre eux les contextes généraux.

Après un premier chapitre théorique et méthodologique, l'ouvrage est structuré en trois parties, qui recouvrent les trois grands moments du rapport de l'Irlande au bouddhisme. La première va $\mathrm{du} \mathrm{VI}^{\mathrm{e}}$ siècle et des premiers textes qui attestent la connaissance de l'existence de cette religion dans l'île, jusqu'au milieu du XIx ${ }^{e}$ siècle. Cette section constitue la toute première tentative visant mettre en forme une histoire de la réception du bouddhisme en Irlande à la période étudiée. L'auteur y démontre que connaissance ne rime en aucun cas avec adoption et conversion en l'absence de facteurs particuliers inconnus avant le milieu du $\mathrm{XIX}{ }^{\mathrm{e}}$ siècle.

La deuxième partie s'intéresse au siècle qui sépare la décennie suivante des années 1960. L'élément nouveau qui va alors déterminer de nouvelles interactions avec le bouddhisme et les premières conversions est le développement de l'Empire 